\title{
Non-Parametric Test
}

National Cancer Institute

\section{Source}

National Cancer Institute. Non-Parametric Test. NCI Thesaurus. Code C53229.

In statistical inference procedures (hypothesis tests and confidence intervals),

nonparametric procedures are those that are relatively free of assumptions about population parameters. 